\title{
Chaos: The speed limiting phenomenon in dynamic atomic force microscopy
}

\author{
Aliasghar Keyvani, ${ }^{1,2}$ Farbod Alijani, ${ }^{1}$ Hamed Sadeghian, ${ }^{2,3, a)}$ Klara Maturova, ${ }^{2}$ \\ Hans Goosen, ${ }^{1}$ and Fred van Keulen ${ }^{1}$ \\ ${ }^{1}$ Department of Precision and Microsystems Engineering, Delft University of Technology, Delft, \\ The Netherlands \\ ${ }^{2}$ Netherlands Organization for Applied Scientific Research, TNO, Delft, The Netherlands \\ ${ }^{3}$ Department of Mechanical Engineering, Eindhoven University of Technology, Eindhoven, The Netherlands
}

(Received 14 August 2017; accepted 21 November 2017; published online 14 December 2017)

\begin{abstract}
This paper investigates the closed-loop dynamics of the Tapping Mode Atomic Force Microscopy using a new mathematical model based on the averaging method in Cartesian coordinates. Experimental and numerical observations show that the emergence of chaos in conventional tapping mode AFM strictly limits the imaging speed. We show that, if the controller of AFM is tuned to be faster than a certain threshold, the closed-loop system exhibits a chaotic behavior. The presence of chaos in the closed-loop dynamics is confirmed via bifurcation diagrams, Poincaré sections, and Lyapunov exponents. Unlike the previously detected chaos due to attractive forces in the AFM, which can be circumvented via simple changes in operation parameters, this newly identified chaos is seemingly inevitable and imposes an upper limit for the closed-loop bandwidth of the AFM. Published by AIP Publishing. https://doi.org/10.1063/1.5000130
\end{abstract}

\section{INTRODUCTION}

The Atomic Force Microscope (AFM) is a versatile instrument for the topography measurement of samples with nanometer resolution. As shown in Fig. 1, the functioning of the AFM is based on measuring the effects of the interactions between a sample surface and a probe. The probe consists of a microcantilever beam and an atomically sharp tip. One of the most popular operation modes is the Tapping Mode AFM (TM-AFM), also known as the amplitude modulation AFM. In this mode, the probe is excited around its fundamental resonance frequency such that its amplitude is set to a so-called free air amplitude. The motion of the cantilever is measured (typically) using an Optical Beam Deflection (OBD) system, and its amplitude is calculated using a Lockin Amplifier (LIA) circuit and a Digital Signal Processing unit (DSP). A set point of the amplitude is reached by bringing the cantilever close to the sample surface. The distance between the cantilever and the sample is adjusted using a fecdback controlled piczoclectric actuator so that the amplitude of the vibration remains constant. While scanning the sample in in-plane directions ( $\mathrm{x}-\mathrm{y}$, hereafter) and keeping the amplitude constant, the control signal is interpreted as the topography of the sample. Moreover, the phase delay of the cantilever provides another image, which is usually interpreted as an indication of surface damping.

In general, a major issue with scanning probe microscopy techniques is their limited imaging speed, and TMAFM is not an exception in this respect. Yet, many of the industrial applications, such as inspection and metrology in semiconductor production lines, demand a high throughput. In fact, different components in the AFM architecture including cantilever, actuators, controller, and the electronic components all have a certain speed limit. Therefore, increasing

\footnotetext{
${ }^{a}$ Electronic mail: hamed.sadeghianmarnani@tno.nl
}

the imaging speed necessitates increasing the bandwidth of every single component as well as optimization of the coupled system. ${ }^{20}$ To enhance the speed of the AFM, many researchers have studied, designed, and characterized a highbandwidth apparatus, ${ }^{2,7,10,19,20,24,29}$ which has led to the improvement of high speed AFMs. Thanks to the advances in precision engineering and fabrication technologies, the high speed AFMs are fast enough, for example, to capture video-rate information from biological processes. ${ }^{2}$ However, further increase of the imaging speed, for example, to cover larger areas and capture faster processes requires substantial improvements of the bandwidth of the cantilever and stability of the closed loop system. To meet these requirements, researchers have suggested to use low-quality factor cantilevers in high-speed AFMs, which has the disadvantage of increasing the Tip-Sample Interaction (TSI) forces. ${ }^{1,28}$ In this research, we aim to understand the main phenomena that are limiting a high-speed AFM from a cantilever-controller coupling point of view.

The requirements for the AFM as a topography measurement tool are twofold: 1) performing a precise and fast raster scanning in the $x-y$ direction, 2) accurate and fast measurement of the local height (h) of the sample in the $\mathrm{Z}$ direction. The latter also determines the maximum speed allowed for the raster scanning i.e., scanning too fast without performing an accurate $\mathrm{Z}$ measurement can cause crash or surface loss. ${ }^{6,14,21}$ Fortunately, linear (or weakly nonlinear) parts such as actuators, sensors, filters, and the ProportionalIntegral (PI) controller do have a cut-off frequency or relaxation time which determines their speed. However, for the strongly nonlinear dynamics of the cantilever interacting with the surface, determining such a limit-frequency is an elusive problem. For example, it is well known that imaging with excessively high scanning speeds can cause imaging artifacts, parachuting effect, surface loss, ${ }^{6}$ and damage. ${ }^{14}$ Yet, the speed limit for which the coupled cantilever- 


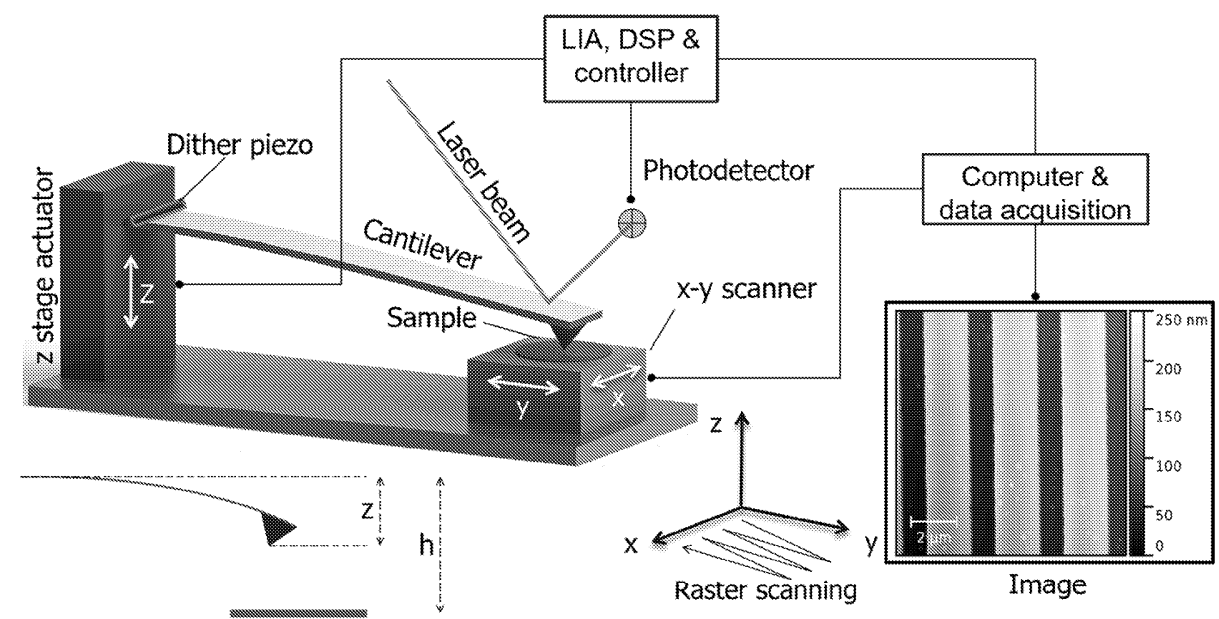

FIG. 1. Schematic view of an AFM, showing the process of imaging the sample. controller combination can precisely follow the surface profile is not well understood.

Another major concern in the AFM which is not extraneous to the speed limit is its closed-loop stability. Due to the coexistence of strongly nonlinear attractive and repulsive Tip-Sample Interaction (TSI) forces, the AFM cantilever exhibits a complex behavior which includes bi-stability and chaos. ${ }^{25}$ In this context, Garcia and San Paulo have presented a comprehensive study which demonstrates the coexistence of two stable regimes at the same time using basin of attractors and experimental results. ${ }^{8}$ Also, researchers have reported the presence of chaos in the AFM as a result of attractive nonlinear van der Waals forces, ${ }^{5,12}$ or excessive adhesion. ${ }^{13}$ This bistability or chaotic behavior indeed causes some artifacts and imaging problems, but it can be eliminated by minimizing the relative effects of the attractive or adhesive forces. One can use stiffer cantilevers, higher material $^{1}$ or environmental damping, ${ }^{28}$ or set a higher free air amplitude (the amplitude of the cantilever far from the sample surface) so that the elastic and repulsive part of the forces become more dominant than the attractive parts. It is also possible to increase the stiffness and damping ratio virtually using a so-called Q-control technique so that the chaotic behavior is eliminated. For example, Ashhab et al. ${ }^{3}$ analyzed the dynamics of the cantilever using Melnikov's method to detect the presence of weak chaos and suggested to use a feedback loop to change the damping ratio. It should be noted that in previous studies, ${ }^{5,12,13}$ the presence of chaos was realized by studying solely the dynamics of the cantilever without addressing the coupled controller-cantilever dynamics. However, we will demonstrate that even if there is no chaotic behavior triggered by the cantilever per se, the controller itself can make the system chaotic, if high gains are applied to achieve fast scanning.

From a control engineering perspective, the cantilever is a part of the Z-stage control unit which ultimately performs a local distance measurement. In steady state situations, and when the repulsive forces are more dominant, the vibration amplitude of the cantilever is approximately equal to the distance between the cantilever and the surface of the sample. Thus, the approximate distance is directly measured via the amplitude signal, if the OBD is calibrated. To retain a constant distance between the cantilever and the surface, a control engineer would intuitively increase the controller gains to force the AFM head to follow the surface faster. However, in practice, this is not possible. From experimental observations, it is well known that there exists an upper bound for the control gains of the system. For higher gains, the amplitude, phase, and the height signals vaguely fluctuate and never reach a steady state response. Consequently, it becomes impossible to capture a relevant image of the sample surface.

In this paper, we investigate the nature of the aforementioned upper bound via a new mathematical model based on an averaging method. The use of the averaging method was essential to incorporate multiple time scales of the AFM dynamics, and also to make the averaged TSI force differentiable for the stability analysis. The results show that the vague fluctuations of the amplitude that appear in the experiments are a result of deterministic chaos. The fact that highspeed (high-gain) controllers induce chaos confirms that the imaging speed of the AFM with the conventional architecture is strictly limited. The presence of chaos in the closedloop system has been confirmed via the bifurcation diagram, Poincaré sections, and Lyapunov exponents.

\section{EXPERIMENTAL OBSERVATIONS}

All the experiments in this section have been performed with a commercial Bruker FastScan AFM with a relatively low-frequency but relatively stiff cantilever (resonance frequency $60 \mathrm{kHz}, \mathrm{Q}$ factor 300 and a spring constant of $1 \mathrm{~N} / \mathrm{m}$ ). All other components of the AFM system are much faster than the cantilever, and consequently, their dynamics can be ignored. To avoid possible chaotic behavior due to attractive or adhesive TSI forces, their relative effects are minimized by choosing a relatively high vibration amplitude, besides having a stiff cantilever (free air amplitude of $150 \mathrm{~nm}$ and amplitude ratio $70 \%$ ).

To demonstrate the effect of control gains, the following two experiments were conducted. First, the cantilever was approached to the surface with integral gain $k_{i}=1$, and proportional gain $k_{p}=5$. In the second experiment, the integral gain was increased to 5 times its previous value, without changing the $x-y$ position or any other setting. Moreover, the 

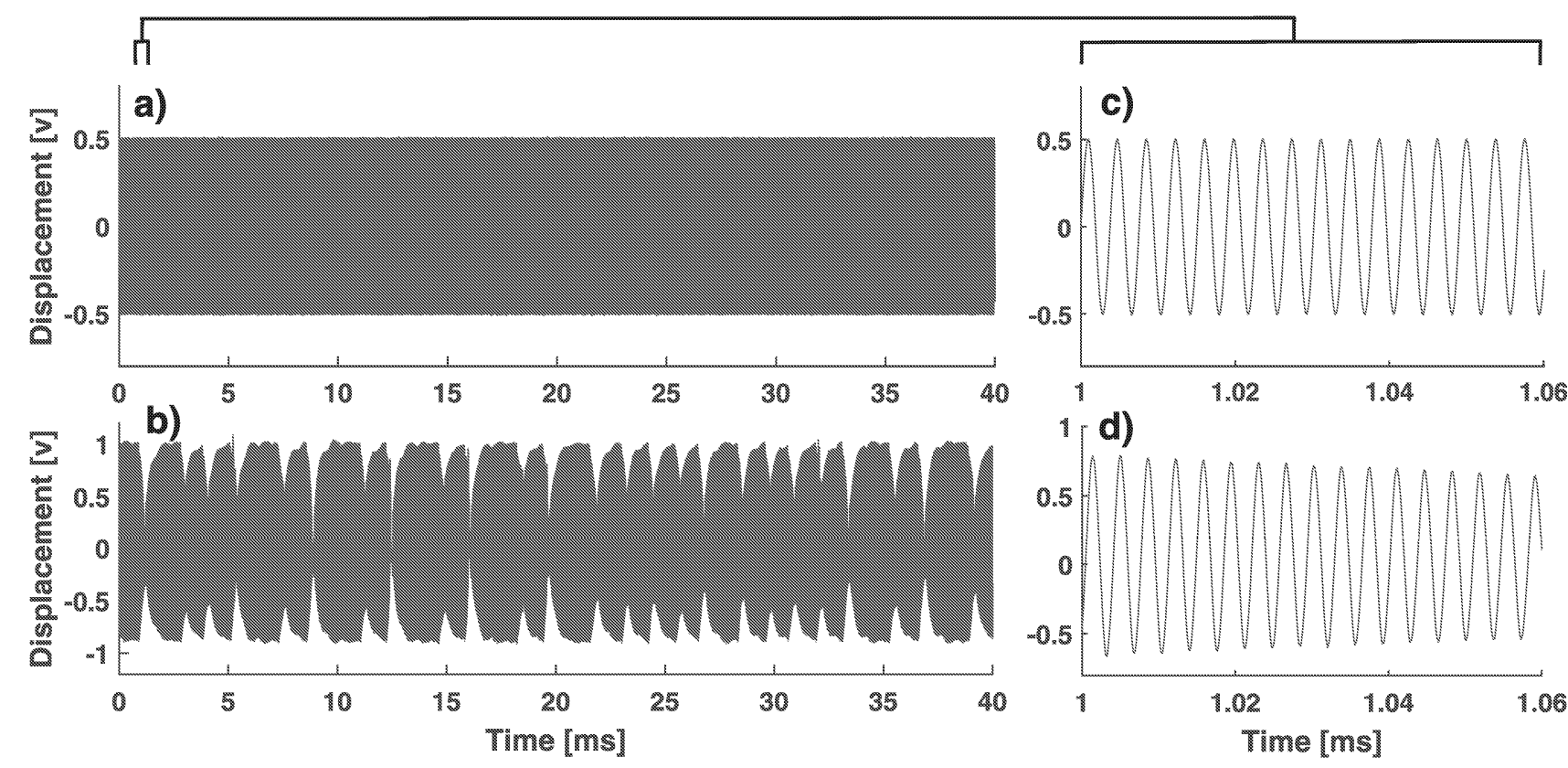

FIG. 2. Measured motion of the cantilever while engaged with the sample surface. (a) Stable with low control gains, (b) aperiodic with high control gains. (c) and (d) show a zoomed version of (a) and (b), respectively.

scan size in both the experiments was set to zero so that only a single point on the sample surface is involved. Figure 2 compares the two cases. For the low control gain, [Figs. 2(a) and 2(c)] the probe is engaged with the surface and is vibrating harmonically. However, for the high control gain, [Figs. 2(b) and 2(d)] the amplitude of the vibration is fluctuating in a non-periodic manner.

To show the effect of integral gain on the imaging performance, Fig. 3 depicts the height image of a silicon dioxide on silicon grating captured with the low and high integral gains.

It can be observed that the image obtained with the higher control gain is unstable due to the aperiodic motion seen in Fig. 2(b). Hence, one can conclude that increasing the measurement bandwidth in the $\mathrm{Z}$ direction by increasing the control gains is not feasible. The nature of this aperiodic motion will be determined in Secs. IV and V.

\section{MATHEMATICAL MODELLING}

In this section, we try to understand the chaotic behavior of the closed-loop AFM system. Therefore, the controller and the cantilever have to be modeled as a coupled system. The coupled system, however, involves two different time scales: A fast time scale for tracking the motion of the cantilever and the tip-sample interactions, and a slow time scale associated with the controller, the amplitude, and the phase signals. In TM-AFM, the cantilever vibrates with a frequency closed to its resonance frequency, and experiences the fast time scale TSI forces. However, within that time scale, no noticeable change in the state of the controller happens. That is, the controller is not affected by the instantaneous motion of the cantilever, but only by the envelope of the motion of the cantilever, which by definition is order(s) of magnitude slower than the cantilever itself. In practice, the Lock-In Amplifier (LIA) separates these two time scales by demodulating the motion signal to its amplitude and phase. ${ }^{18}$ The details of the LIA are not within the scope of this paper; however, it is important to note that there does not exist any transfer function or any linear approximation for the LIA. Hence, to incorporate the functionality of LIA in the closed-loop model, one should either solve the nonlinear equations in time domain, or alternatively, derive a demodulated model. The extreme nonlinearity of the LIA also makes it impossible to conduct a frequency domain stability analysis, such as those based on Nyquist or Nichols diagrams.

Although it is theoretically possible to model the AFM system without the separation of time scales, and including all the nonlinearities and vibration modes of the cantilever, ${ }^{4,26}$ such a model would be complex and computationally

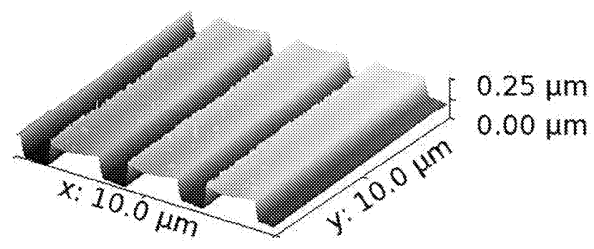

a) Stable regime

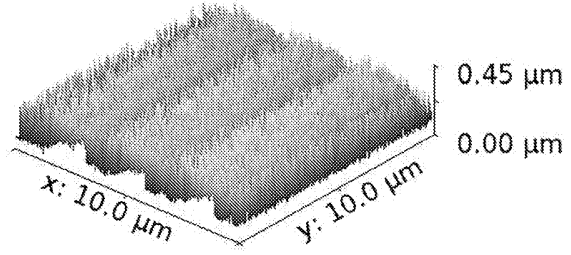

b) Chaotic regime
FIG. 3. AFM imaging results of a silicon dioxide on silicon grating sample in two situations: (a) stable with low control gains and (b) unstable with high control gains. 
inefficient. Considering that the LIA only measures the amplitude and phase of a single harmonic component of the signal, the higher modes of the cantilever are not visible to the LIA, and consequently, do not affect the closed-loop dynamics. Alternatively, we use a single degree-of-freedom (DOF) model of the AFM cantilever and derive a demodulated model for the closed-loop system which already incorporates the functionality of the LIA. Also, since the chaotic behavior studied in this paper is independent of the attractive or adhesive forces, we limit the TSI force model to the Hertzian contact force. Similar multiple time scale problems have been solved with different techniques in the literature $;^{15,23}$ however, in AFM research, the slow dynamic models are limited to virial theory, ${ }^{22}$ and the periodic averaging method. ${ }^{11}$ The periodic averaging method eliminates the short time scale from the dynamics of the system by applying a Fourier operator to the governing differential equation. However, in previously reported models, ${ }^{11,22}$ the second-order derivatives of the amplitude and phase have been ignored which degrades the accuracy of the transient analysis.

A non-dimensional single-DOF model of the cantilever can be formulated as

$$
\begin{gathered}
\ddot{z}+\xi \dot{z}+z=F_{d} \cos (\omega t)+F_{t s}, \\
F_{t s}=-\beta(z-h)_{H_{0}}^{3 / 2},
\end{gathered}
$$

where $z$ denotes the nondimensional deflection of the cantilever and $h$ is the nondimensional distance between the tip and the sample at the zero deffection configuration; both are normalized with respect to the free air amplitude. In Eq. (1a), the dot denotes differentiation with respect to dimensionless time which is defined by normalizing the time with respect to the inverse of the eigenfrequency of the resonator. In addition, $\xi$ is the damping ratio, and $\omega$ is the normalized excitation frequency, which is also normalized with respect to the resonance frequency of the cantilever ( $\omega \approx 1$ for TM-AFM). $F_{d}$ is the dimensionless force equivalent to the acoustic excitation from the dither piezoelectric actuator, and $\beta$ represents the Hertzian stiffness of the contact area between the tip and the sample. The subscript $H_{0}$ represents a Heaviside function used to model the discontinuity due to indentation.

Equation (1) can be cast into the state space representation as follows:

$$
\left\{\begin{array}{c}
\dot{q}_{1} \\
\dot{q}_{2}
\end{array}\right\}=\left\{\begin{array}{c}
-\xi q_{1}-q_{2}-\beta\left(q_{2}-h\right)_{H_{0}}^{3 / 2}+F_{d} \cos (\omega t) \\
q_{1}
\end{array}\right\}
$$

where $q_{1}=\dot{z}$ and $q_{2}=z$. To separate the short and long time scales and incorporate the functionality of the LIA, we assume that the state variables in Eq. (2) are amplitude modulated harmonic signals as

$$
\begin{aligned}
& q_{1}=\Re\left(\bar{q}_{1} e^{j \omega t}\right)=\Re\left(\left(x_{1}+j x_{2}\right) e^{j \omega t}\right), \\
& q_{2}=\Re\left(\bar{q}_{2} e^{j \omega t}\right)=\Re\left(\left(x_{3}+j x_{4}\right) e^{j \omega t}\right),
\end{aligned}
$$

in which the $\bar{q}_{i}(i=1,2)$ represents the amplitude and phase of $q_{i}$ in polar coordinates and $x_{k}(k=1, \ldots, 4)$ are the Cartesian representations of $\bar{q}_{i}, \Re$ is the real operator and $j^{2}=-1$.

Substituting Eq. (3) in (2), taking the derivatives and rearranging for $\overline{\dot{q}}$ yields

$$
\left\{\frac{\overline{\dot{q}_{1}}}{\dot{\bar{q}}_{2}}\right\} e^{j \omega t}=\left\{\begin{array}{l}
-j \omega \bar{q}_{1} e^{j \omega t}-\xi \bar{q}_{1} e^{j \omega t}-\bar{q}_{2} e^{j \omega t}-\beta\left(\bar{q}_{2} e^{j \omega t}-h\right)_{H_{0}}^{3 / 2}+F_{d} \cos (\omega t) \\
-j \omega \bar{q}_{2} e^{j \omega t}+\bar{q}_{1} e^{j \omega t}
\end{array}\right\} .
$$

Applying the periodic averaging method to Eq. (4), which is, multiplying by $e^{-j \omega t}$ and integrating over a period $\left(\langle\ldots\rangle=\int_{t_{0}}^{t_{0}+\frac{2 \pi}{\omega}} \ldots e^{-j \omega t} d t\right)$, gives the demodulated governing differential equations for the cantilever as

$$
\left\{\begin{array}{c}
\overline{\dot{q}_{1}} \\
\dot{\dot{q}_{2}}
\end{array}\right\}=\left\{\begin{array}{c}
-j \omega \bar{q}_{1}-\xi \bar{q}_{1}-\bar{q}_{2}+\left\langle F_{t s}\right\rangle+F_{d} \\
-j \omega \bar{q}_{2}+\bar{q}_{1}
\end{array}\right\}
$$

in which the $\left\langle F_{t s}\right\rangle=-\int_{t_{0}}^{t_{0}+\frac{2 \pi}{\omega}} \beta\left(\bar{q}_{2} e^{j \omega t}-h\right)_{H_{0}}^{3 / 2} e^{-j \omega t} d t$, is the first Fourier component of the TSI force that can be calculated as

$$
\left\langle F_{t s}\right\rangle=\beta \sqrt{A I}\left(\frac{h}{A}\right) \bar{q}_{2}
$$

where

$$
I(\zeta)=\int_{0}^{2 \pi}(\cos (\tau)-\zeta)_{H 0}^{3 / 2} \cos (\tau) d \tau
$$

$A=\left|\bar{q}_{2}\right|=\sqrt{x_{3}^{2}+x_{4}^{2}}$ is the amplitude of the cantilever, and $\zeta$ and $\tau$ are dummy variables. The integral function $I(\zeta)$ can be calculated separately which eliminates the need for tracking the short time scale. In this manner, instead of the TSI force which had to be tracked in the short time scale, only the periodic average (i.e., the first Fourier component) of the TSI force is considered, that instead varies slowly in time.

In this model, it is assumed that every other component of the AFM, such as actuators and electronics are infinitely faster than the cantilever. Thus, here, we only couple an ideal PI controller to the modulated model of the cantilever given by Eq. (5). As such, an additional state variable is introduced which relates the amplitude error $\left(A_{\text {set }}-A\right)$, to the height signal $h$, with the integral and proportional actions as follows:

$$
\begin{gathered}
\dot{x_{5}}=k_{i}\left(A_{s e t}-A\right), \\
h=x_{5}+k_{p}\left(A_{\text {set }}-A\right),
\end{gathered}
$$

where $A_{s e t}$ is the set-point amplitude. In Eq. (8), the integrator accumulates the error in the internal state of the controller $\left(x_{5}\right)$ and the output of the controller is a weighted sum of the instantaneous error (with proportional gain $k_{p}$ ) and the 
accumulated error (with the integral gain $k_{i}$ ). Expanding the real and imaginary parts of Eq. (4), the closed-loop model of the AFM can be written as

$$
\begin{aligned}
\dot{x_{1}}= & -\xi x_{1}+\omega x_{2}-x_{3} \\
& -\beta \sqrt[4]{x_{3}^{2}+x_{4}^{2}} I_{1}\left(\frac{k p\left(A_{s e t}-\sqrt{x_{3}^{2}+x_{4}^{2}}\right)+x_{5}}{\sqrt{x_{3}^{2}+x_{4}^{2}}}\right) x_{3}+f_{d} \\
\dot{x_{2}}= & -x_{1} \omega-\xi x_{2}-x_{4} \\
& -\beta \sqrt[4]{x_{3}^{2}+x_{4}^{2}} I_{1}\left(\frac{k_{p}\left(A_{s e t}-\sqrt{x_{3}^{2}+x_{4}^{2}}\right)+x_{5}}{\sqrt{x_{3}^{2}+x_{4}^{2}}}\right) x_{4} \\
\dot{x_{3}}= & x_{1}+\omega x_{4}, \\
\dot{x_{4}}= & x_{2}-\omega x_{3}, \\
\dot{x_{5}}= & k_{i}\left(A_{\text {set }}-\sqrt{x_{3}^{2}+x_{4}^{2}}\right)
\end{aligned}
$$

Equation (9) shows a dynamic relationship between the amplitude, phase, and height signals in frames of the Cartesian coordinates, which evolve with the slow time scale. In Sec. IV, we shall use this model to simulate different TM-AFM scenarios and detect the nonperiodic behavior observed experimentally in Sec. II.

\section{CLOSED-LOOP RESPONSE OF AFM}

Many parameters ranging from deflection sensitivity of the cantilever to linearity and static gain of voltage amplifiers play a role in the total performance of the system. However, it is outside the scope of this paper to quantitatively determine the maximum stable PI gains. Thus, we limit ourselves to a qualitative demonstration of the chaos in the coupled system, and ignore the dynamics of actuators and also dismiss the static gains and sensitivities of the system.
Figure 4 shows the amplitude and height signals during an approach scenario for three different integral gain settings. In this scenario, the dither piezo actuator and the controller turn on at time zero. For all three cases, the proportional gain is set to $k_{p}=0.01$, the initial distance between the probe and the sample is 5 times the free air amplitude and the set-point amplitude is 0.5 times the free air amplitude. All numerical results refer to a cantilever with quality factor 100 , which is excited at its resonance frequency $(\omega=1)$, and is engaged to a sample with a nondimensional Hertzian modulus of $\beta=2000$. These parameters roughly correspond to the realistic imaging conditions.

For all three cases, initially, the amplitude is less than the set-point, and the height signal increases to above 5 units. Then, as soon as the amplitude reaches its set point value, the controller starts to reduce the height. The height reduction continues until the amplitude has decreased to its setpoint. In Fig. 4(b), the integral gain has been increased to $k_{i}=0.01$. In this case, the cantilever reaches the surface faster than in Fig. 4(a), and thus, the surface loss effects, such as the parachuting effect, ${ }^{6}$ have been reduced. However, the amplitude and height signals fluctuate for a longer time. Figure $4(\mathrm{c})$ shows the same scenario with an even higher controller gain $\left(k_{i}=0.1\right)$. Figure 5 shows an experimental counterpart of Fig. 4(c), measured using a commercially available AFM (Bruker FastScan) and a standard tapping mode cantilever (MPP 22120). The spring constant, resonance frequency, and the Quality factor of the cantilever were measured using the thermal calibration technique as $0.65 \mathrm{n} / \mathrm{m}, 49.01 \mathrm{kHz}$, and 180 , respectively. The excitation frequency was chosen such that the free air amplitude is $5 \%$ less than the maximum amplitude (default for AFM). The free air amplitude was set at $80 \mathrm{~nm}$ and the setpoint amplitude was set at $50 \mathrm{~nm}$. An AFM training sample (Fused
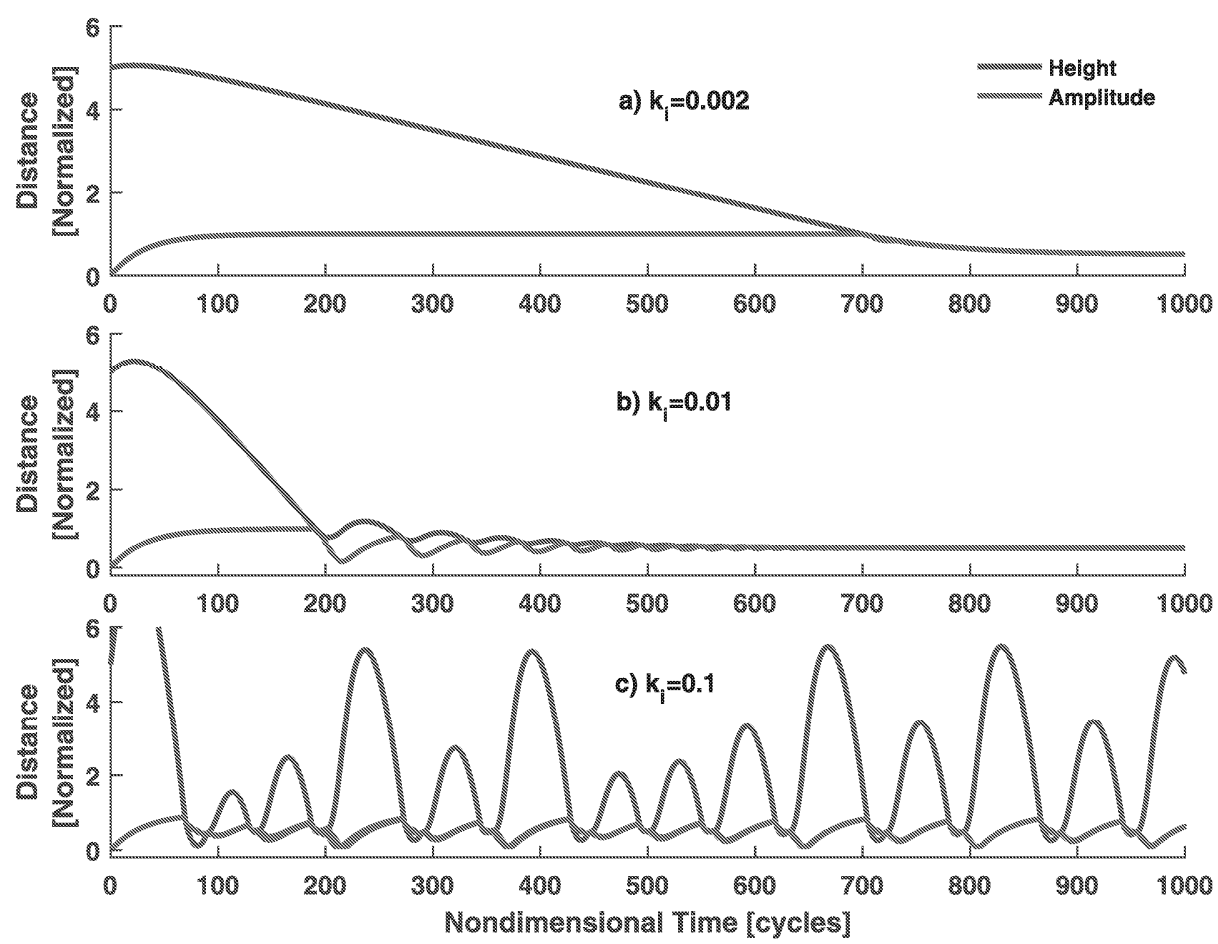

FIG. 4. Simulated approach process with three different integral gains. 


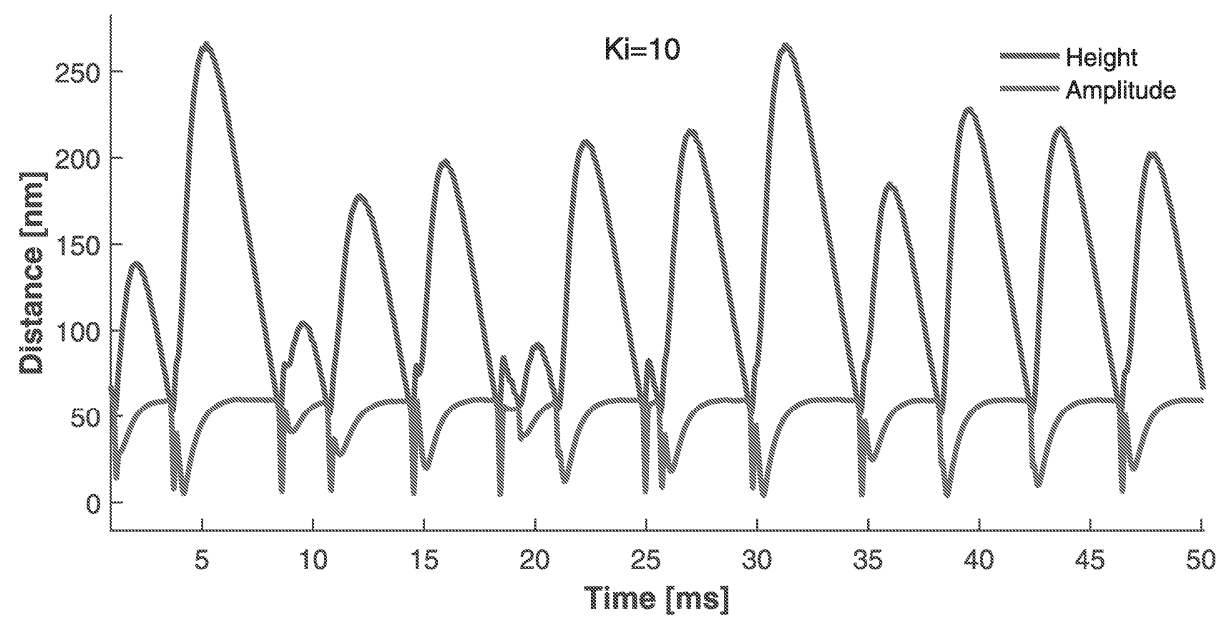

FIG. 5. Measured amplitude and phase of the TM-AFM with high control gain $(\mathrm{Ki}=10)$.
Silica) was used as the substrate, and the scanning range was set at zero to avoid any sample related deterioration as much as possible. As it can be seen, both the numerical and experimental results suggest that for high control gains, the amplitude and height signals drop and increase in a nonpredictable manner which potentially could be due to chaos.

\section{CHAOS}

In this section, the nonlinear dynamics of the system described by Eq. (9) will be examined to confirm that the unusual response observed experimentally is indeed deterministic chaos. In order to identify the peculiar characteristics of chaos, first, a bifurcation diagram of the system is obtained to study the effect of the integral gain on the behavior of the system. Next, the response of the system in the phase space will be studied which shows the attractors of the system corresponding to the different regions of the bifurcation diagram. As another indication of chaos, the nonperiodicity of the response is studied using frequency spectrum and Poincaré sections. Finally, the sensitivity to initial conditions is demonstrated via time histories and Lyapunov exponents.
As mentioned earlier, many parameters can affect the response of the system; yet, here, we restrict ourselves to the effects of the integral gain of the controller because of its direct relation to the bandwidth of the closed-loop system. Figure 6 shows the bifurcations of the amplitude in the engaged configuration when the integral gain is increased. For small integral gains, there exists only one amplitude value (equal to the set-point amplitude) to which the system will eventually settle. Increasing the integral gain further, there is a region $\left(k_{i}=0.018-0.02\right)$ where two values for the amplitude coexist. This part of the bifurcation diagram implies a periodic fluctuation of the amplitude between the two values. In practice, this area is still moderately tolerable for imaging applications. This is because the two amplitudes are close to one another, and thus, the fluctuation between the two does not induce a considerable error signal for the controller. However, increasing the integral gain further, clouds of points are seen in the bifurcation diagram $\left(k_{i}>0.024\right)$ representing a chaotic motion.

To experimentally determine the upper limit of the integral gain, and verify the presence of bifurcation and chaos presented in Fig. 6, we repeated the same experiment as used

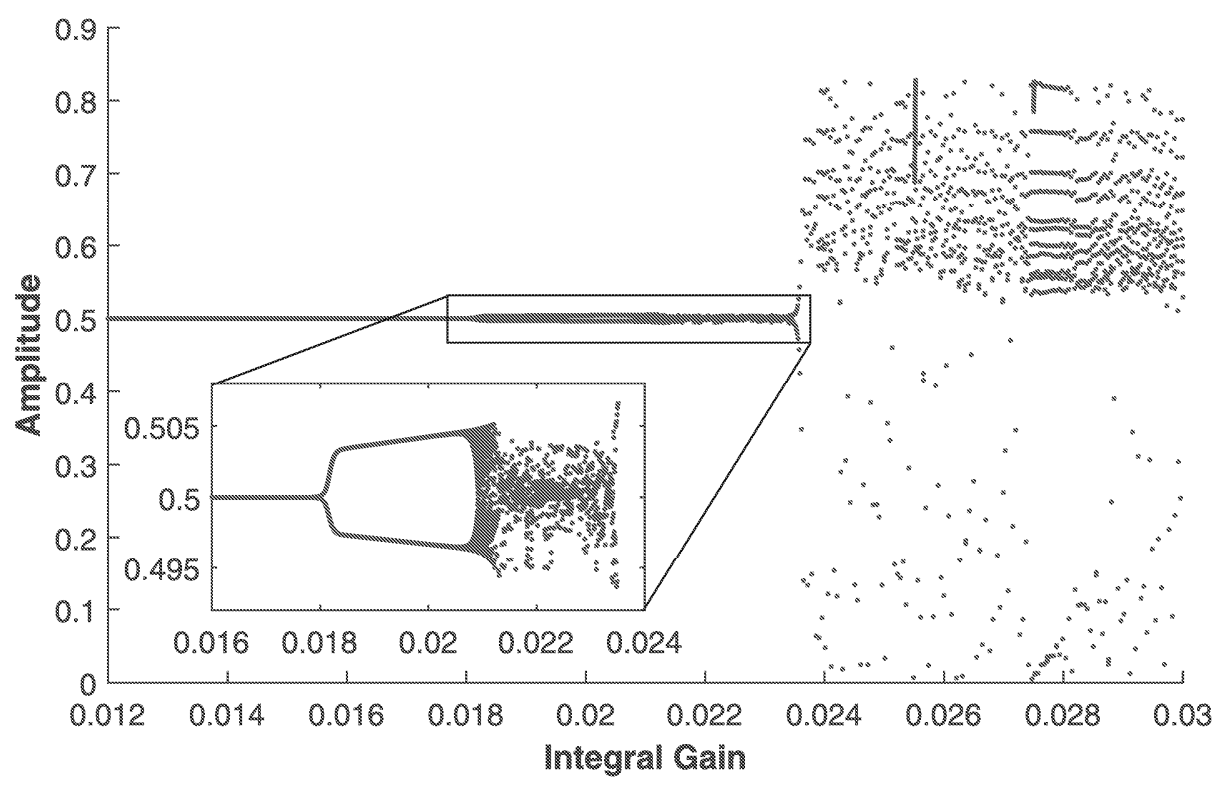

FIG. 6. Bifurcation diagram of the TM-AFM, the steady state amplitude versus the integral gain. 


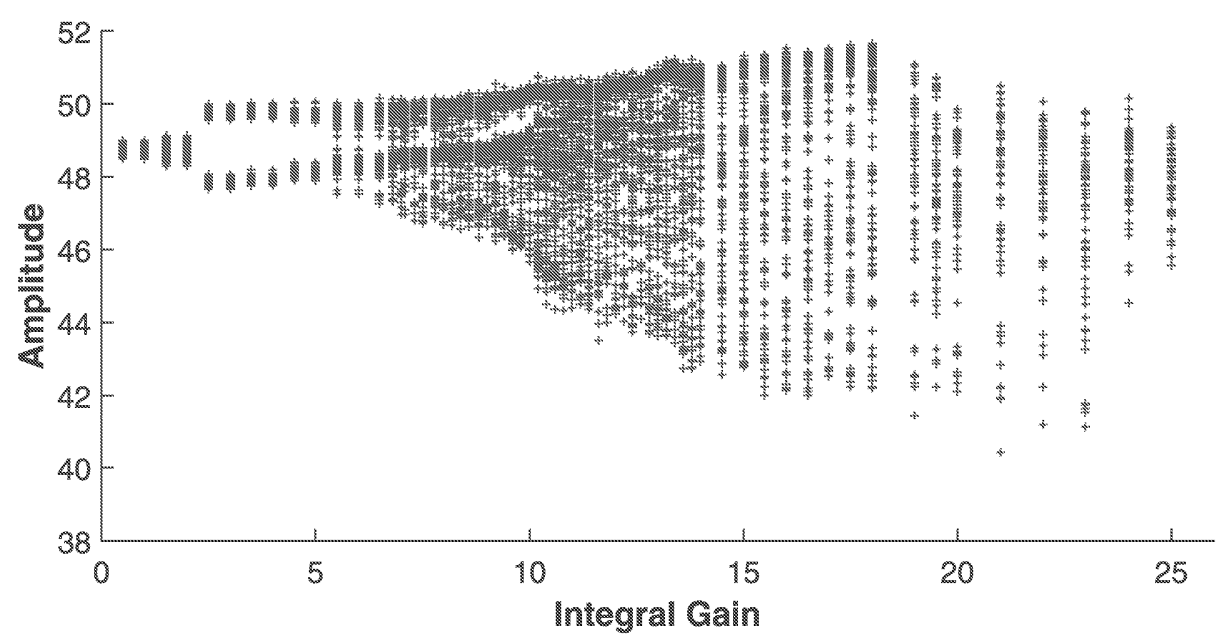

FIG. 7. Experimental bifurcation diagram of the TM-AFM, the amplitude versus integral gain.

for Fig. 5, but with control gains ranging between 1 and 25 . For all of the experiments, the proportional gain was 1 . To plot the bifurcation diagram in Fig. 7, the amplitude values were measured on a hypersurface in state space on which the phase value is equal to its steady-state value (where $k_{i}=1$ ). As it can be seen, for intermediate integral gains $\left(k_{i}=2.5-6.5\right)$, two values for the amplitude signal coexist, whereas, for higher control gains, a cluster of randomly distributed points were measured, which indicates $k_{i}=6.5$ as the upper limit of the control gain. Note that this limit is measured for the specific cantilever and experimental conditions, and does not provide a universal value by any means. Since different physical parameters of the system affect its stability (not only the integral gain), a more thorough investigation should be conducted to identify the chaotic and nonchaotic set of parameters.
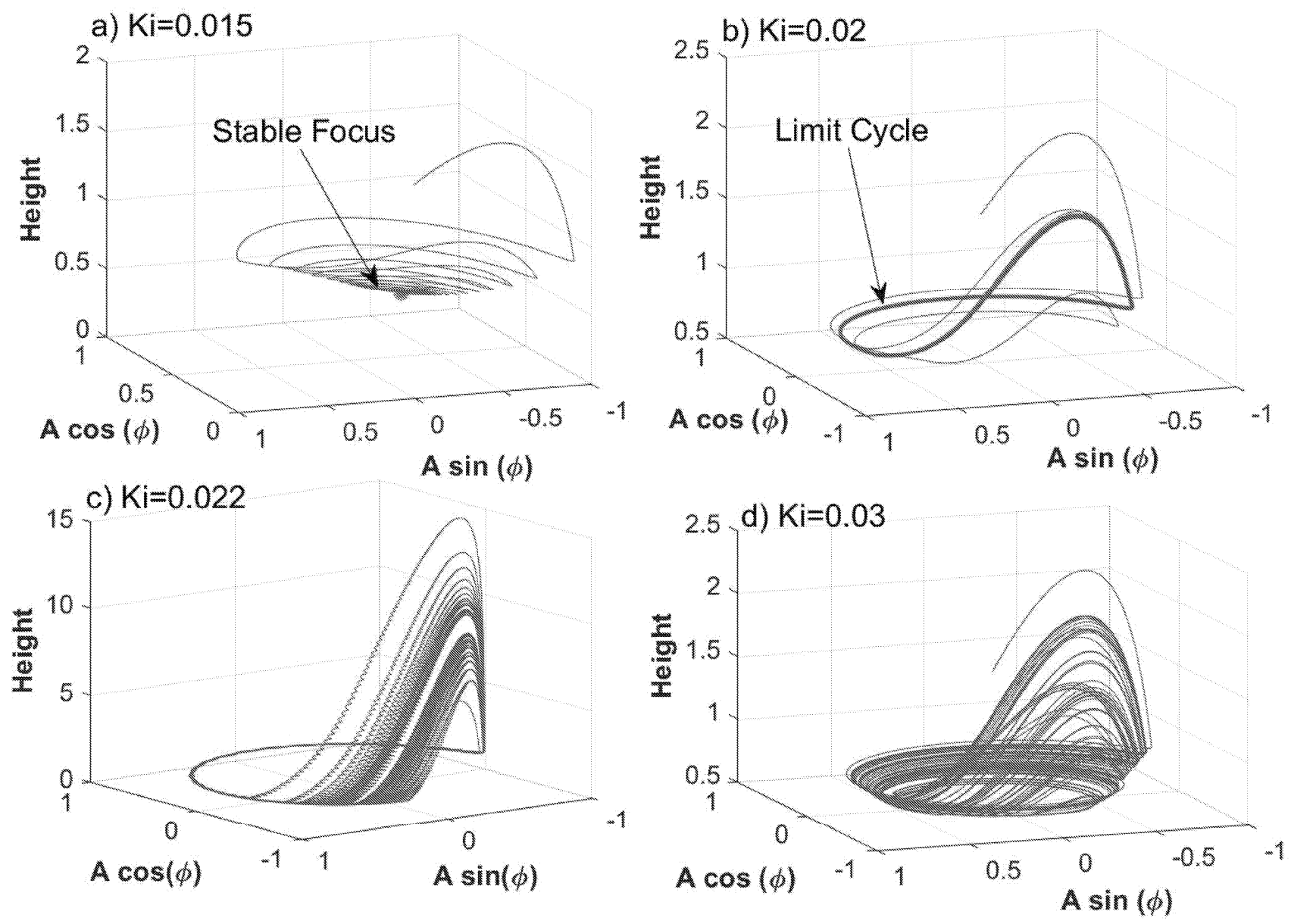

FIG. 8. A projection of the attractors of the TM-AFM on the amplitude, phase, and height space. (a) Stable focus, (b) stable limit cycle, and (c) and (d) strange attractor. All the values are normalized with respect to the free air amplitude. 
Here, it should be noted that the theoretical results in Sec. IV are achieved with a completely deterministic model, even though the cluster of points in Fig. 6 indicate a randomlike response. In order to show the deterministic nature of the irregular fluctuations in Figs. $4(\mathrm{c})$, and 6, the geometry of the attractors of the system has to be studied in the phase space. Figure 8 shows the projection of the attractors onto the $x_{3}, x_{4}, x_{5}$ space for three different integral gains within each of the sections of Fig. 6. As it can be seen in Fig. 8(a), when the integral gain is small (e.g., $k_{i}=0.015$ ), the steadystate response of the system approaches a stable focus [the orange point in Fig. 8(a)], which has the amplitude equal to the set-point amplitude and corresponding phase and height. For the second case $\left(k_{i}=0.02\right)$, as the effects of the initial conditions vanish, the states of the system gradually approach a limit cycle, shown in orange in Fig. $8(\mathrm{~b})$, and remain on that closed-loop. However, for a slightly higher value of the integral gain $\left(k_{i}=0.022\right)$, the limit cycle is nonexistent, and the system never repeats itself. This nonexistence of a steady-state response in Fig. 8(c) and d is associated with the strange attractor, which is an indication of chaos. Figure 9 shows the experimental counterpart of Fig. 8 , in which similar behavior for all the four regimes is visible.

To further investigate the behavior of the system in the chaotic regime, we present a Poincaré section of the state space considering two different Poincaré surfaces. Figure
10 (a) shows the Poincaré section of the system on a constant-height surface, and it shows the amplitude and the phase with which the cantilever leaves the surface every time that it disengages. Figure 10(b) shows the amplitude and height while the phase reaches its expected value for the steady state case. The cluster of individual points in Figs. 10 (a) and 10(b) denotes the complexity of the strange attractors.

The complexity of the chaotic motion in the frequency domain translates to a wide spectrum of frequency components that resemble the frequency spectrum of noise. Figure 11 shows the Discrete Fourier Transform (DFT) of all five state variables of the system. As it can be seen, the attractor of the system contains a wide band of frequency components. The absence of clearly distinct peaks in the DFT (Fig. 11) indicates that the signal is not periodic.

Perhaps the most important characteristic of a chaotic system is its sensitivity to initial conditions. The long-term response of a chaotic system is extremely sensitive to the changes in initial conditions, meaning that two trajectories with very similar initial conditions might end up in very different final configurations. ${ }^{16}$ One simple test of initial condition dependency for TM-AFM is shown in Fig. 12. The same approach curve in Fig. $4(\mathrm{c})$ is simulated with two different but close initial heights $[h(0)=5.0000$ and $<h(0)=5.0001]$. As it can be seen in Fig. 12, the two trajectories are almost identical at the beginning. However,
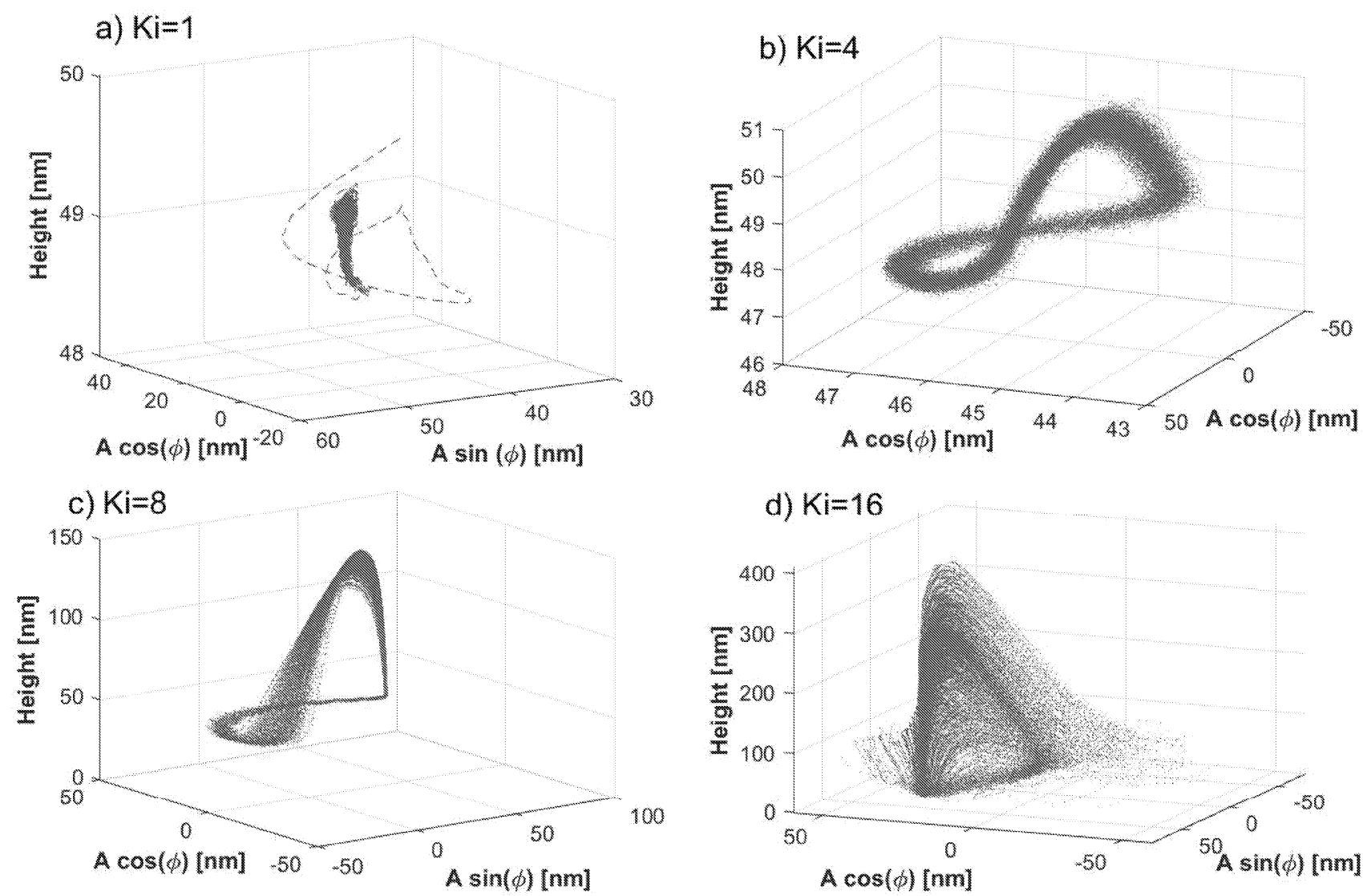

FIG. 9. Measured projection of the attractors of the TM-AFM on the amplitude, phase, and height space: (a) stable focus, (b) stable limit cycle, and (c) and (d) strange attractors. 

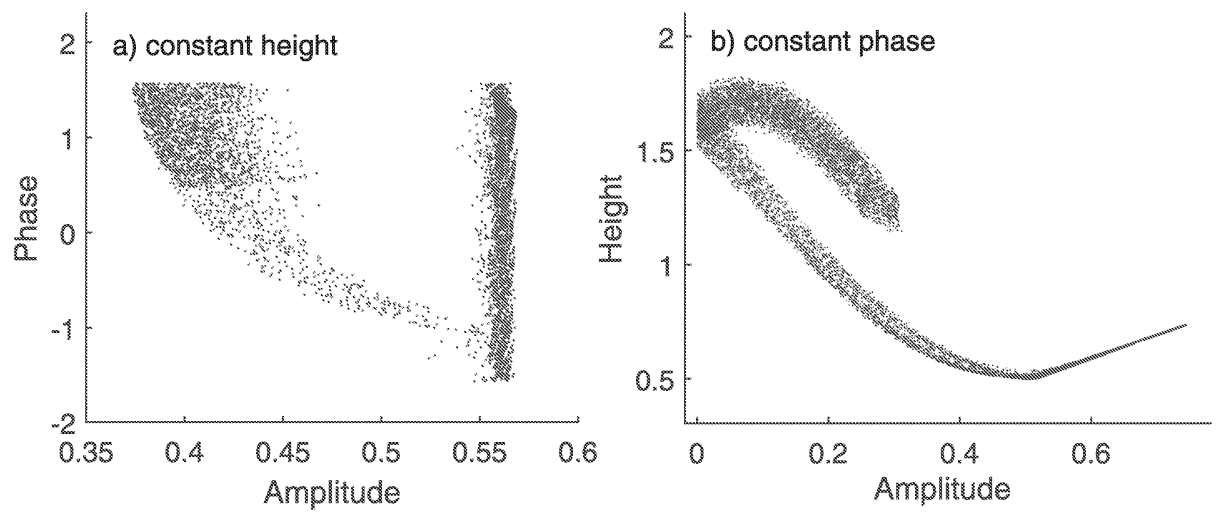

FIG. 10. Poincaré sections of the closed-loop TM-AFM on the hypersurfaces perpendicular to (a) constant height and (b) constant phase.

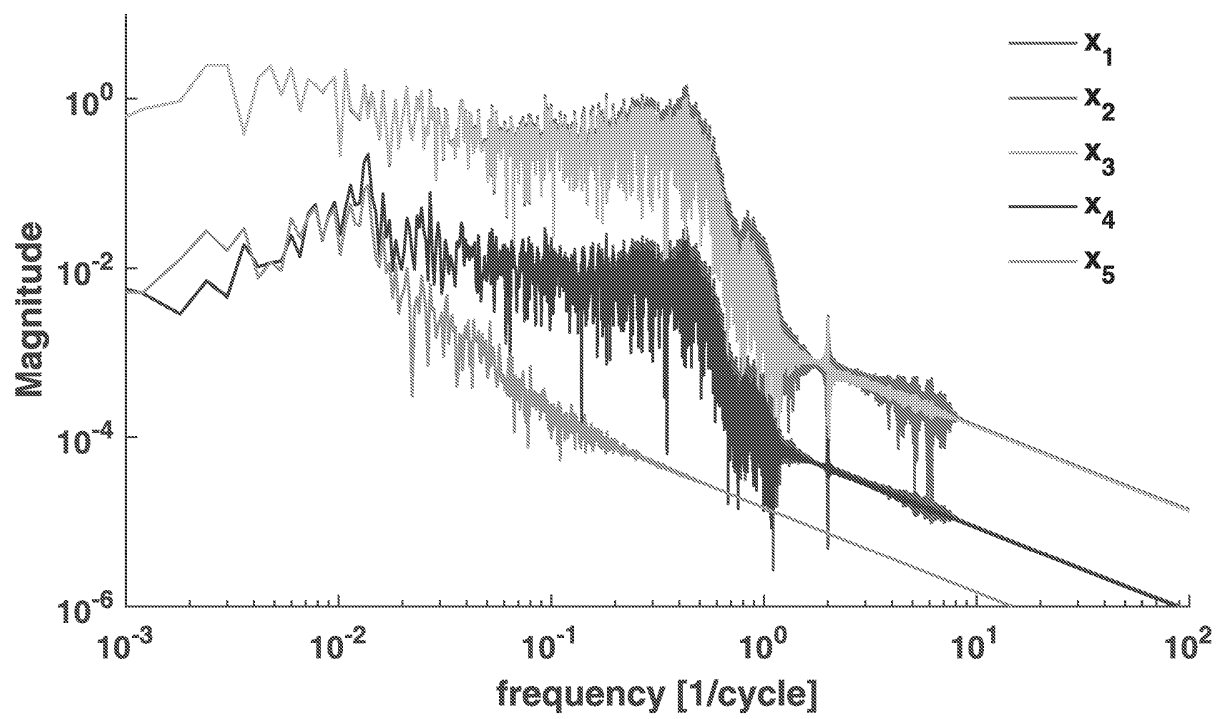

FIG. 11. Fourier transform of the states of closed-loop AFM in chaotic situations.

after about 300 vibration cycles, they start to deviate from each other.

A very important and solid indicator of sensitivity to initial conditions in dynamics systems is the Lyapunov exponents of the attractor of the system. ${ }^{17,27}$ Lyapunov exponents of dynamic systems show the average rate of exponential divergence of any two trajectories along their attractors. To determine the Lyapunov exponents for the closed-loop TMAFM system, we adopt the method of QR decomposition presented by Geist et al. ${ }^{9}$ Figure 13 demonstrates these
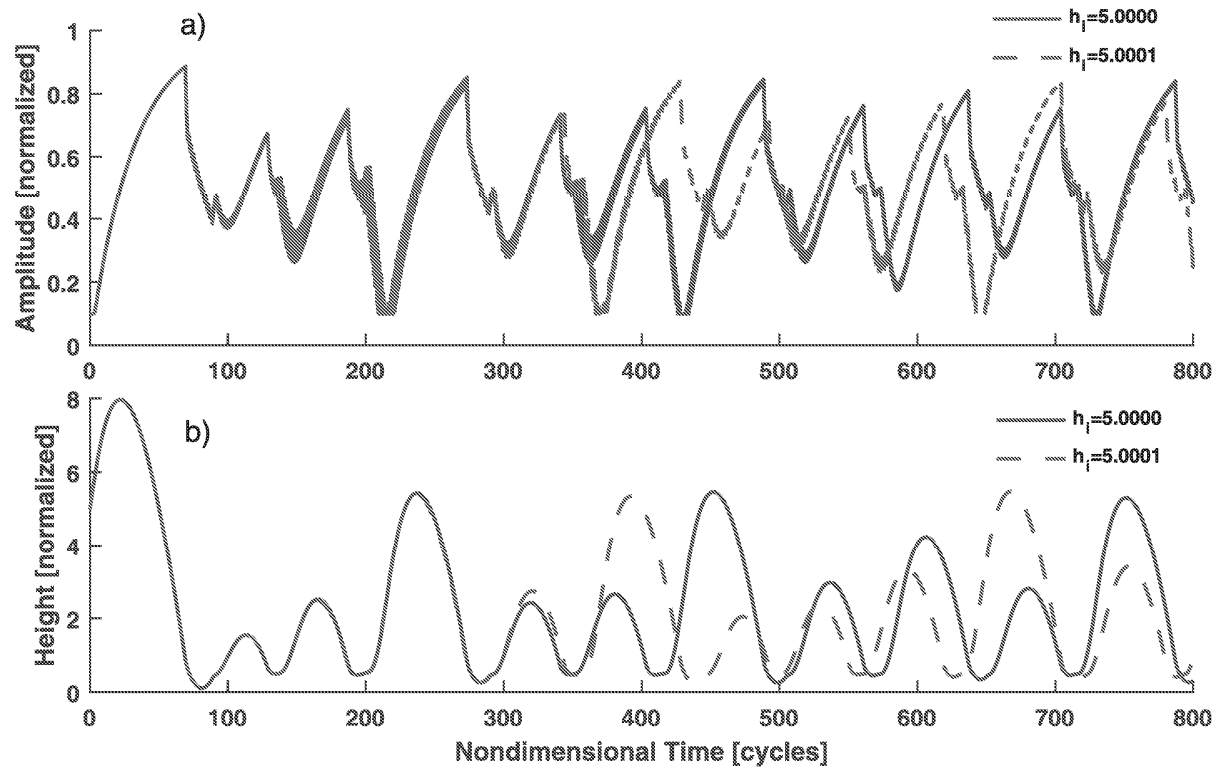

FIG. 12. Simulated approach process with high controller gains for two different but similar initial conditions: (a) amplitude and (b) height. 


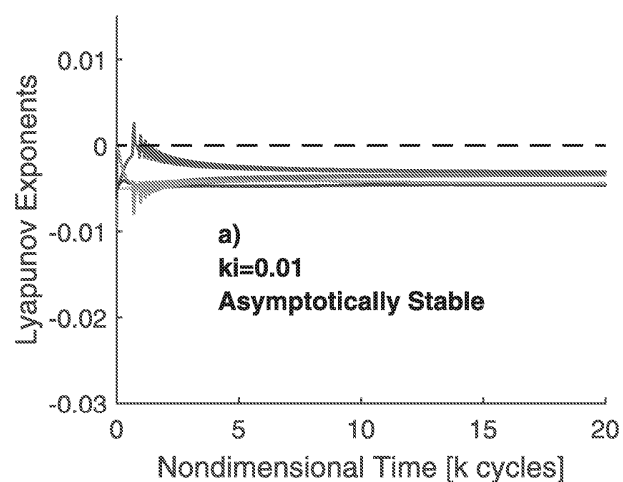

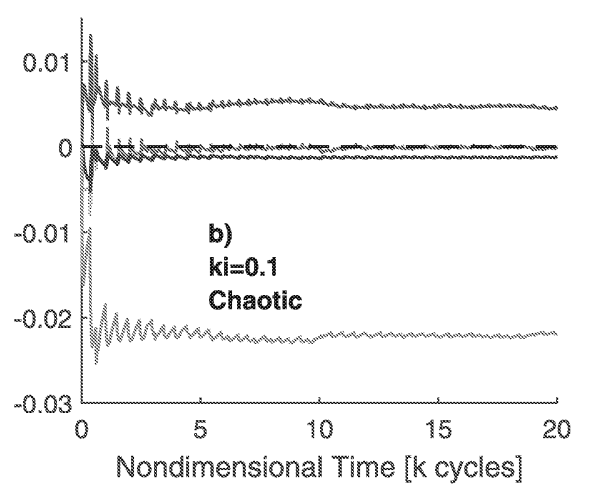

FIG. 13. Lyapunov exponents of the closed-loop TM-AFM in case (each color shows one Lyapunov exponent). (a) The control gains are low and the system is stable. (b) The control gains are high and the system is chaotic.
Lyapunov exponents for two different cases of low and high integral gains. As it can be seen in Fig. 13(a), for the case of low control gains, all the Lyapunov exponents of the system converge to a negative value which refers to the asymptotically stable attractor (the set-point). However, for the case with high control gains [Fig. 13(b)], the largest Lyapunov exponent is positive, which shows that any small deviation from the attractor will exponentially grow in the state space. The summation and all the other Lyapunov exponents (except the highest one) are negative which show that the system does not experience hyper-chaos or a global instability.

\section{CONCLUSIONS}

Experimental results with the tapping mode AFM show that it is not possible to image with control gains higher than a certain amount. Such a limit in the control gains restricts the imaging speed of the AFM. To investigate the origin of this limit, a new nonlinear dynamics model for the AFM was presented using an averaging method in the Cartesian coordinates. The model incorporates the coupled dynamics of the cantilever and the controller, and demonstrates that the tapping mode AFM will exhibit a chaotic behavior with high controller gains. The corresponding attractors, Poincaré sections, and Lyapunov exponents formally confirmed the presence of chaos in TM-AFM. Unlike the previously presented routes to chaos in the AFM, the presented route of chaos does not depend on the attractive or adhesive tip-sample forces and limits the speed of TM-AFM, even when the controller and the actuators are selected ideally in terms of the bandwidth. This phenomenon should be taken into account in the design of high-speed AFM and to avoid it, either the cantilever dynamics or the architecture of the closed-loop system should be modified.

\section{ACKNOWLEDGMENTS}

This work was supported by the Netherlands Organization for Applied Scientific Research, TNO, Early Research Program 3D Nanomanufacturing.

1J. D. Adams, B. W. Erickson, J. Grossenbacher, J. Brugger, A. Nievergelt, and G. E. Fantner, "Harnessing the damping properties of materials for high-speed atomic force microscopy," Nat. Nanotechnol. 11(2), 147-151 (2016).
${ }^{2}$ T. Ando, T. Uchihashi, and T. Fukuma, "High-speed atomic force microscopy for nano-visualization of dynamic biomolecular processes," Prog. Surf. Sci. 83(7), 337-437 (2008).

${ }^{3}$ M. Ashhab, M. Salapaka, M. Dahleh, and I. Mezić, "Melnikov-based dynamical analysis of microcantilevers in scanning probe microscopy," Nonimear Dyn. 20(3), 197-220 (1999).

${ }^{4}$ H.J. Butt, B. Cappella, and M. Kappl, "Force measurements with the atomic force microscope: Technique, interpretation and applications," Surt. Sci. Rep. 59(1), 1-152 (2005).

5. H. Cantrell and S. A. Cantrell, "Bifurcation, chaos, and scan instability in dynamic atomic force microscopy," J. Appl. Phys. 119(12), 125308 (2016).

${ }^{6}$ T. De, P. Agarwal, D. R. Sahoo, and M. V. Salapaka, "Real-time detection of probe loss in atomic force microscopy," Appl. Phys. Let?. 89(13), $133119(2006)$.

${ }^{7}$ A. Fleming, B. Kenton, and K. K. Leang, "Bridging the gap between conventional and video-speed scanning probe microscopes," Ultramicroscopy $110(9), 1205-1214(2010)$.

${ }^{8} \mathrm{R}$. Garcia and A. San Pavlo, "Dynamics of a vibrating tip near or in intermittent contact with a surface," Phys. Rev. B 61(20), R13381 (2000).

${ }^{9} \mathrm{~K}$. Geist, U. Parlitz, and W. Lauterborn, "Comparison of different methods for computing lyapunov exponents," Prog. Theor. Phys. 83(5), 875-893 (1990).

${ }^{10}$ R. Herfst, B. Dekker, G. Witvoet, W. Crowcombe, D. de Lange, and H. Sadeghian, "A miniaturized, high frequency mechanical scanner for high speed atomic force microscope using suspension on dynamically determined points," Rev. Sci. Instrum. 86(11), 113703 (2015).

${ }^{11} \mathrm{~S}$. Hu, "Nonlinear dynamics and force spectroscopy in dynamic atomic force microscopy," Ph.D. thesis (Purdue University, 2007).

${ }^{12}$ S. Hu and A. Raman, "Chaos in atomic force microscopy," Phys. Rev. Lett. 96(3), 036107 (2006).

${ }^{13}$ F. Jamitzky, M. Stark, W. Bunk, W. Heckl, and R. Stark, "Chaos in dynamic atomic force microscopy," Nanotechnology 17(7), S213 (2006).

${ }^{14}$ A. Keyvani, H. Sadeghian, H. Goosen, and F. van Keulen, "Transient tipsample interactions in high-speed afm imaging of $3 \mathrm{~d}$ nano structures," in SPIE Advanced Lithography (International Society for Optics and Photonics, 2015), p. 94242Q.

${ }^{15}$ C. Kuehn, Multiple Time Scale Dynamics (Springer, 2015), Vol. 191

${ }^{16}$ E. N. Lorenz, "Deterministic nonperiodic flow," I. Atmos. Sci. 20(2), 130-141 (1963).

${ }^{17}$ F. C. Moon, Chaotic and Fractal Dynamics: Introduction for Applied Scientists and Engineers (John Wiley \& Sons, 2008).

${ }^{18}$ A. V. Oppenheim, A. S. Willsky, and S. H. Nawab, Signals and Systems, 2nd ed. (Prentice-Hall, Inc., Upper Saddle River, NJ, USA, 1996).

${ }^{19}$ H. Sadeghian, R. Herfst, B. Dekker, J. Winters, T. Bijnagte, and R. Rijnheek, "High-throughput atomic force microscopes operating in parallel," Rev. Sci. Instrum, 88(3), 033703 (2017).

${ }^{20}$ H. Sadeghian, R. Herfst, J. Winters, W. Crowcombe, G. Kramer, T. van den Dool, and M. H. van Es, "Development of a detachable high speed miniature scanning probe microscope for large area substrates inspection," Rev. Sci. Instrum. 86(11), 113706 (2015).

${ }^{21}$ H. Sadeghian, T. C. van den Dool, Y. Uziel, and R. B. Or, "High-speed afm for $1 \mathrm{X}$ node metrology and inspection: Does it damage the features?," in SPIE Advanced Lithography (International Society for Optics and Photonics, 2015), p. 94240Q.

${ }^{22}$ A. San Paulo and R. García, "Tip-surface forces, amplitude, and energy dissipation in amplitude-modulation (tapping mode) force microscopy," Phys. Rev. B 64(19), 193411 (2001). 
${ }^{23}$ J. A. Sanders, F. Verhulst, and J. Murdock, Averaging Methods in Nonlinear Dynamical Systems (Springer-Verlag, New York, 2007).

${ }^{24}$ S. Kuiper and G. Schitter, "Improving the imaging speed of AFM with modern control techniques," in Control Technologies for Emerging Micro and Nanoscale Systems, Lecture Notes in Control and Information Sciences Vol. 413, edited by E. Eleftheriou and S. O. R. Moheimani (Springer, Berlin, Heidelberg, 2011).

${ }^{25}$ R. W. Stark, "Bistability, higher harmonics, and chaos in afm," Mater. Today 13(9), 24-32 (2010).

${ }^{26}$ R. W. Stark, G. Schitter, M. Stark, R. Guckenberger, and A. Stemmer, "State-space model of freely vibrating and surface-coupled cantilever dynamics in atomic force microscopy," Phys. Rev. B 69(8), 085412 (2004).

${ }^{27}$ S. H. Strogatz, Nonlinear Dynamics and Chaos: With Applications to Physics, Biology, Chemistry, and Engineering (Westview Press, 2014).

${ }^{28}$ E. M. H. Van and H. Sadeghian, "Euv blank defect and particle inspection with high-throughput immersion afm with $1 \mathrm{~nm} 3 \mathrm{~d}$ resolution," in SPIE Advanced Lithography (nternational Society for Optics and Photonics, 2016), p. $97782 \mathrm{Z}$.

${ }^{29} \mathrm{Y}$. Wang, X. Hu, L. Xu, and X. Hu, "Improving the scanning speed of atomic force microscopy at the scanning range of several tens of microm meters," Ultramicroscopy 124, 102-107 (2013). 